\title{
NED416, a novel synthetic Sirt1 activator, promotes cutaneous wound healing via the MAPK/Rho pathway
}

\author{
HUSSAIN MUSTATAB WAHEDI ${ }^{1,2}$, JAE KHYUNG CHAE $^{2}$, LALITA SUBEDI ${ }^{2}$, \\ MIN CHEOL KANG ${ }^{2}$, HYUNKYUNG CHO ${ }^{3}$, SANGHEE KIM ${ }^{3}$ and SUN YEOU KIM ${ }^{2,4,5}$
}

\author{
${ }^{1}$ Department of Biological Sciences, National University of Medical Sciences, C/O Military Hospital, Rawalpindi, \\ Punjab 46000, Pakistan; ${ }^{2}$ College of Pharmacy, Gachon University, Incheon 21936; ${ }^{3}$ College of Pharmacy, \\ Seoul National University, Seoul 08826; ${ }^{4}$ Gachon Institute of Pharmaceutical Science, Gachon University, Incheon 21936; \\ ${ }^{5}$ Gachon Medical Research Institute, Gil Medical Center, Incheon 21547, Republic of Korea
}

Received October 5, 2019; Accepted March 6, 2020

DOI: $10.3892 /$ ijmm.2020.4564

\begin{abstract}
Cutaneous wound healing is a highly complex biological process involving major events such as cell migration, angiogenesis, and tissue development. Sirtuin 1 (Sirt1) and its regulators have been suggested to play a role in cell migration and tissue repair. The aim of the present study was to determine the effects of a novel Sirt1 activator, the piper amide derivative (E)-3-(2,4-dichlorophenyl)- $N$-phenylacrylamide, also known as NED416, on cutaneous wound healing. The effects of NED416 on Sirt1 activity, Sirt1 expression, and angiogenesis were measured in skin and endothelial cells (epidermal keratinocytes, dermal fibroblasts and vascular endothelial cells) using a Sirt1 activity assay kit, western blot analysis and tube formation assays, respectively. The effects of NED416 on the rate of wound closure and collagen deposition were measured via $\mathrm{H} \& \mathrm{E}$ staining and Masson's trichrome staining, respectively. Levels of migration-related [Rac1, cell division cycle 42 (Cdc42) and $\alpha$-p21-activated kinase] and mitogen-activated protein kinase (MAPK) signaling pathway proteins were measured in hairless mice via western blot analysis. NED416 significantly increased Sirt1 activity in dermal fibroblasts and epidermal keratinocytes to a greater extent than resveratrol, leading to increased cell migration and angiogenesis through Rac1/Cdc42 and ERK/JNK activation. Furthermore, NED416 accelerated wound closure, macrophage infiltration, and epithelium and collagen formation in vivo. The present study demonstrated a role of Sirt1 in cutaneous wound healing, and suggested that NED416 as a Sirtl activator is more potent than resveratrol in promoting wound healing
\end{abstract}

Correspondence to: Professor Sun Yeou Kim, College of Pharmacy, Gachon University, 191 Hambakmoero, Yeonsu-gu, Incheon 21936, Republic of Korea

E-mail: sunnykim@gachon.ac.kr

Key words: regeneration, sirtuin 1, skin, wound healing, drug discovery through Rac1/Cdc42 and MAPK signaling without toxicity, thus serving as a promising candidate for treatment.

\section{Introduction}

Wound healing is one of the most complex biological processes and affects the whole organism, in addition to the site of injury (1). The cutaneous wound healing process can be divided into six phases: Hemostasis; inflammation; proliferation and migration; angiogenesis; re-epithelialization; and synthesis or remodeling (2-4); the three broad stages of the healing process are inflammation, proliferation and remodeling. The inflammation phase is characterized by the presence of tumor necrosis factor- $\alpha$ and interleukin (IL) -6 , which are released by keratinocytes; this release leads to the infiltration of macrophages and neutrophils to the wound site (5). This infiltration ensures the regulation of wound healing in the phases to follow via proinflammatory cytokines such as IL-1 $\beta$ and IL-6, as well as growth factors such as epidermal growth factor, transforming growth factor $\beta-1$ (TGF- $\beta 1$ ) and platelet-derived growth factor, which are released by macrophages (6). In the next phase, known as the proliferation phase, the release of critical cytokines and growth factors mediate key events such as angiogenesis, re-epithelialization and endothelial proliferation (7). Polarization and migration of skin keratinocytes then lead to stratification and differentiation, resulting in wound contraction (8). Fibroblasts and keratinocytes also play a vital role in this migration process (9). The migration of fibroblasts and keratinocytes is initiated by intracellular polarization induced via critical proteins such as Rac1, cell division cycle 42 (Cdc42) and $\alpha$-p21-activated kinase ( $\alpha$-PAK) (10-12). In the final stage of remodeling, TGF- $\beta 1$ mediates the degradation of granular tissue, and a scar containing substantial quantities of type I collagen and immature blood vessels is formed $(13,14)$.

Sirtuins (Sirts) are class III histone deacetylases that regulate numerous biological processes and diseases through the deacetylation of histone and non-histone targets, including aging, inflammation and cancer (15). Among the seven members of the Sirt family of proteins, Sirtl is the most studied and characterized; Sirt1 plays critical roles in various 
important diseases and conditions, such as inflammation (16), oxidative damage (17), apoptosis (18), diabetes (19) and aging (20). Due to its diverse range of targets and functions, certain studies have also elucidated the possible role of Sirt1 in wound healing and cell migration (21-25).

Our previous studies focused on identifying novel Sirt1 activators and evaluating their potential for skin wound healing and regeneration $(26,27)$. In the present study, a novel synthetic activator of Sirt1, $(E)-3$-(2,4-dichlorophenyl)- $N$-phenylacrylamide (NED416; Fig. S1), was investigated for its effects on Sirt1 regulation and the process of cutaneous wound healing.

\section{Materials and methods}

Cell culture. Human umbilical vein endothelial cells (HUVECs; cat. no. CRL-1730 ${ }^{\mathrm{TM}}$ ) and normal human dermal fibroblasts (NHDFs; cat. no. PCS-201-012 ${ }^{\mathrm{TM}}$ ) were purchased from the American Type Culture Collection, while HaCaT epidermal keratinocytes were provided by Professor Tae Yoon Kim from the College of Medicine of The Catholic University of Korea. All the cell lines used in this study were tested for mycoplasma contamination and used in experiments between passage numbers 5 and 10 . HaCaT cells were cultured in high-glucose DMEM (Thermo Fisher Scientific, Inc.) with $10 \%$ fetal bovine serum (FBS) and $1 \%$ penicillin-streptomycin. HUVECs were cultured in M200 medium (Invitrogen; Thermo Fisher Scientific, Inc.) supplemented with 20\% FBS and $1 \%$ low-serum growth supplement (Invitrogen; Thermo Fisher Scientific, Inc.). NHDFs were cultured in fibroblast medium (ScienCell Research Laboratories, Inc.). Cultures were at all times incubated in a humidified atmosphere at $37^{\circ} \mathrm{C}$ and $5 \% \mathrm{CO}_{2}$ unless stated otherwise.

Preparation of NED416. A piper amide derivative, NED416, was synthesized according to a previously reported method (28), purified by silica gel column chromatography and characterized. The spectral data obtained were consistent with previously reported values (29). ${ }^{1} \mathrm{H}-\mathrm{NMR}\left(300 \mathrm{MHz}, \mathrm{CDCl}_{3}\right.$ ): $\delta 8.17$ (br s, $1 \mathrm{H}), 8.00(\mathrm{~d}, J=15.6 \mathrm{~Hz}, 1 \mathrm{H}), 7.65(\mathrm{~d}, J=7.8 \mathrm{~Hz}, 2 \mathrm{H})$, $7.48(\mathrm{~d}, J=8.4 \mathrm{~Hz}, 1 \mathrm{H}), 7.38(\mathrm{~d}, J=1.8 \mathrm{~Hz}, 1 \mathrm{H}), 7.29$ (t, $J=7.8 \mathrm{~Hz}$, 2H), 7.15 (dd, $J=8.0,8.6 \mathrm{~Hz}, 1 \mathrm{H}), 7.08$ (t, $J=7.2 \mathrm{~Hz}, 1 \mathrm{H}), 6.67$ $(\mathrm{d}, J=15.6 \mathrm{~Hz}, 1 \mathrm{H}) .{ }^{13} \mathrm{C}-\mathrm{NMR}\left(100 \mathrm{MHz}, \mathrm{CD}_{3} \mathrm{OD}\right): \delta 165.7$, 139.8, 137.1, 137.0, 136.4, 133.1, 130.9, 129.9 (2C), 129.8, 128.9, 125.8, 125.5, 121.2 (2C; Fig. S2). HRMS (fast atom bombardment): Calculated for $\mathrm{C}_{15} \mathrm{H}_{11} \mathrm{Cl}_{2} \mathrm{NO}[(\mathrm{M})+] 292.0296$, found 292.0299 .

Cell treatment. Cells at $80 \%$ confluence were rinsed and treated with 1,5 , or $10 \mu \mathrm{M}$ NED416 solutions prepared and diluted in dimethyl sulfoxide (DMSO; Thermo Fisher Scientific, Inc.) and serum-free medium, respectively, after washing the cells twice with PBS (PAA Laboratories GmbH; GE Healthcare).

Sirt1 activity measurement. The Sirt1 activity assay was performed in triplicate by using a SIRT1 Direct Fluorescent Screening Assay kit (Cayman Chemical Company). Briefly, $25 \mu 1$ of assay buffer was added to each well of a 96-well plate followed by $5 \mu \mathrm{l}$ of Sirt1, $5 \mu \mathrm{l}$ of NED416 or resveratrol solution, and $15 \mu \mathrm{l}$ of Sirtl substrate solution. Background wells were treated with solvent but not with Sirt1.
Western blotting. Treated cells were harvested and lysed with lysis buffer (150 mM NaCl, 1\% NP-40, 0.5\% sodium deoxycholate, $0.1 \%$ SDS and $50 \mathrm{mM}$ Tris-Cl). The concentration of proteins in the supernatants of lysates was determined using a Bio-Rad Protein Assay (Bio-Rad Laboratories, Inc.), with bovine serum albumin (SERVA Electrophoresis $\mathrm{GmbH}$ ) as the standard. Protein samples $(30 \mu \mathrm{g})$ were subjected to SDS-PAGE on $8-15 \%$ polyacrylamide gels and transferred to nitrocellulose membranes. Membranes were blocked for $1 \mathrm{~h}$ at room temperature with a $5 \%(\mathrm{w} / \mathrm{v})$ non-fat milk solution in TBS- $0.05 \%$ Tween-20. Membranes were then subjected to overnight incubation at $4^{\circ} \mathrm{C}$ with the solutions of primary antibodies against Sirt1 (1:1,000; cat. no. sc-15404; Santa Cruz Biotechnology, Inc.), p53 and acetylated p53 (1:1,000; cat. nos. 9282 and 2570; Cell Signaling Technologies, Inc.), $\alpha$-tubulin (1:1,000; cat. no. SAB3501072; Sigma-Aldrich; Merck KGaA), Rac1 and phosphorylated (p)-Rac1 (1:1,000; cat. nos. 2465 and 2461; Cell Signaling Technologies, Inc.), Cdc42 and p-Cdc42 (1:1,000; cat. nos. 2462 and 2461; Cell Signaling Technologies, Inc.), $\alpha$-PAK (1:1,000; cat. no. sc-166887; Santa Cruz Biotechnology, Inc.), ERK and p-ERK (1:1,000; cat. nos. sc-271291 and sc-136521; Santa Cruz Biotechnology, Inc.), JNK and p-JNK (1:1,000; cat. nos. sc-1648 and sc-6254; Santa Cruz Biotechnology, Inc.), and p38 and p-p38 (1:1,000; cat. nos. sc-136210 and sc-166182; Santa Cruz Biotechnology, Inc.), followed by incubation at room temperature for $2 \mathrm{~h}$ with horseradish peroxidase conjugated anti-rabbit and anti-mouse secondary antibodies (1:500; cat. nos. sc-2357 and sc-516102; Santa Cruz Biotechnology, Inc.). Bands were visualized using a solution comprised of luminal (cat. no. 42586.A.01) and hydrogen peroxide (cat. no. 42585.B.01; both SERVA Electrophoresis $\mathrm{GmbH}$ ) in a 1:1 mixture. The membrane was incubated with this solution for $1 \mathrm{~min}$, then bands were visualized using a chemiluminescence detection system (Bio-Rad Laboratories, Inc.); protein expression was quantified using Alpha View SA Version 3.4.0.0 software (ProteinSimple, Inc.).

MTT assays. To determine cell viability, $1 \times 10^{5} \mathrm{HaCaT}$ cells, which were seeded in 6-well plates or 40-mm culture dishes, were treated with 1,5 or $10 \mu \mathrm{M}$ solutions of NED416 prepared in serum-free medium. The medium was removed after $24 \mathrm{~h}$, and $2 \mathrm{ml}$ of $500 \mu \mathrm{g} / \mathrm{ml}$ MTT solution was added. The cells were then incubated at $37^{\circ} \mathrm{C}$ for $1 \mathrm{~h}$. Then, MTT was replaced with $1 \mathrm{ml}$ of DMSO, and the plates were incubated on a shaker for $30 \mathrm{~min}$ at room temperature. The absorbance at $570 \mathrm{~nm}$ was determined using a microplate reader (Molecular Devices LLC).

Small interfering RNA (siRNA) knockdown. HaCaT cells seeded in 96-well plates ( $60 \%$ confluence) were transfected with $100 \mathrm{nM}$ (final concentration) of Sirtl siRNA or scramble siRNA (Bioneer Corporation) dissolved in serum-free media with an equal volume of a 1.4-1.5\% solution of Lipofectamine ${ }^{\circledR}$ (Invitrogen; Thermo Fisher Scientific, Inc.) prepared in serum-free medium. Cells were then incubated for $6 \mathrm{~h}$ before washing with PBS and then subjected to the cell migration assay. The independent sequences used for silencing Sirt1 were as follows: Sequence 1, sense, 5'-ACUUUGCUGUAA CCCUGUA(dTdT)-3' and antisense, 5'-UACAGGGUUACA GCAAAGU(dTdT)-3'; sequence 2, sense, 5'-AGAGUUGCC ACCCACACCU(dTdT)-3' and antisense, 5'-AGGUGUGGG 
Table I. Criteria to evaluate the histological score of wound healing.

\begin{tabular}{lll}
\hline Score & \multicolumn{1}{c}{ Epidermal and dermal regeneration } & Granulation tissue thickness \\
\hline \pm 1 & Little epidermal and dermal organization & Thin granulation layer \\
\pm 2 & Moderate epidermal and dermal organization & Moderate granulation layer \\
\pm 3 & Complete remodeling of dermis and epidermis & Thick granulation layer \\
\pm 4 & & Very thick granulation layer
\end{tabular}

A

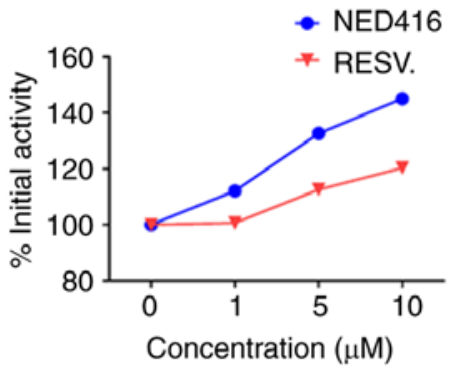

B

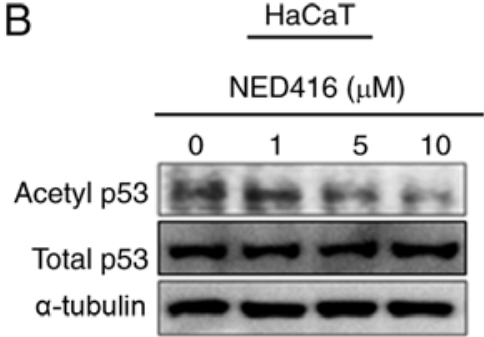

C
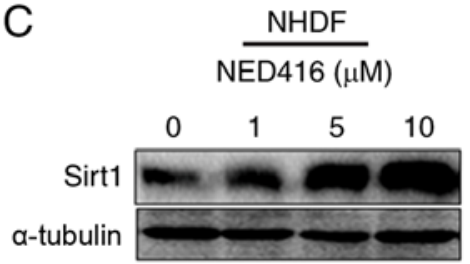

$\mathrm{D}$

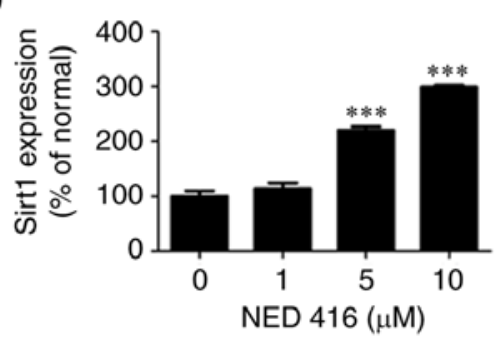

E
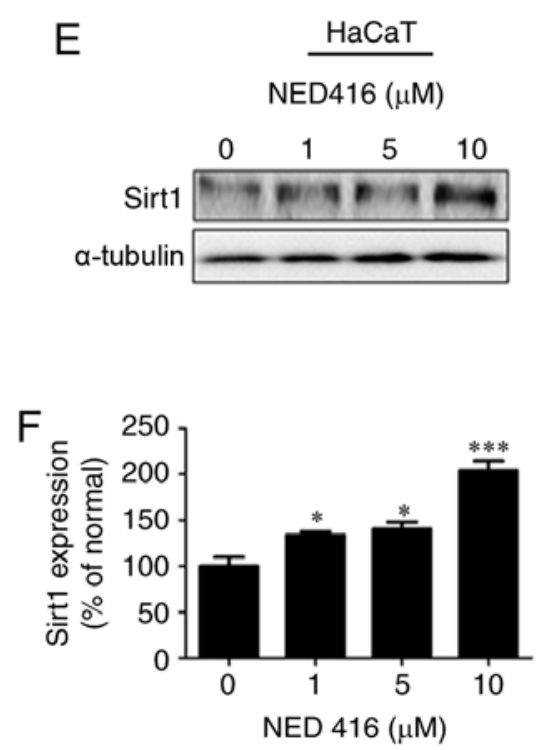

Figure 1. NED416 increases Sirt1 expression and activity in skin cells. (A) NED416 significantly enhanced the enzymatic activity of Sirt1 in a concentration-dependent manner. (B) Acetylation of p53 after NED416 treatment. (C) NHDF cells treated with different concentrations of NED416 for 24 h showed a dose-dependent increase in Sirt1 expression in western blot analysis. (D) Quantification of Sirt1 expression in NHDF cells following NED416 treatment. (E) HaCaT cells treated with different concentrations of NED416 for $24 \mathrm{~h}$ exhibited a dose-dependent increase in the expression of Sirt1 in western blot analysis. (F) Quantification of Sirt1 expression in HaCaT cells following NED416 treatment. Data are presented as the mean \pm SEM. ${ }^{*} \mathrm{P}<0.05,{ }^{* * *} \mathrm{P}<0.001$ vs. $0 \mu$ M. NED416, (E)-3-(2,4-dichlorophenyl)- $N$-phenylacrylamide; Sirt1, sirtuin 1; NHDF, normal human dermal fibroblasts; resv., resveratrol.

UGGCAACUCU(dTdT)-3'. The sequence for the scramble siRNA was as follows: Sense, 5'-AGAGUUCAAAAGCCC UUCA(dTdT)-3' and antisense, 5'-UGAAGGGCUUUUGAA CUCU(dTdT)-3'.

Cell migration assays. HaCaT cells were seeded in 96-well plates 1 day before the assay. A wound was created in the cell layer using a Wound Maker ${ }^{\mathrm{TM}}$ tool (Essen BioScience, Ltd.). Wounded cells were incubated at $37^{\circ} \mathrm{C}$ for $24 \mathrm{~h}$ in the presence of NED416 or serum-free medium. In the experiments demonstrating the effect of the ERK pathway on cell migration, the wounded cells were pretreated with $10 \mu \mathrm{M}$ of ERK inhibitors SP600125, SB203580 and U0126 (cat. nos. S5567, S8307 and U120; all Sigma-Aldrich; Merck $\mathrm{KGaA}$ ) for $1 \mathrm{~h}$ prior to treatment with NED416. Images of the cells were captured every $4 \mathrm{~h}$ using IncuCyte $\mathrm{ZOOM}^{\circledR}$ (magnification, $\mathrm{x} 40$; Essen BioScience, Ltd.) to monitor the effects of NED416 on cell migration.

Endothelial cell tube formation assay. HUVECs were cultured in M200 medium (Invitrogen; Thermo Fisher Scientific, Inc.) supplemented with 20\% FBS and 1\% low-serum growth supplement (Invitrogen; Thermo Fisher Scientific, Inc.) at $37^{\circ} \mathrm{C}$ and $5 \% \mathrm{CO}_{2}$. Each well of the 96-well plates was coated with $50 \mu \mathrm{l}$ of Matrigel matrix and incubated at $37^{\circ} \mathrm{C}$ for $30 \mathrm{~min}$. HUVECs were suspended in serum-free medium containing $1 \mathrm{X}$ LGS, and $1 \times 10^{6}$ HUVECs were seeded in each well of the 96-well plates pretreated with Matrigel matrix with the vehicle (M200) or NED416 for $18 \mathrm{~h}$. Tube formation was captured using an inverted microscope (magnification, $\mathrm{x} 40$; Nikon Corporation).

Ethical approval. All procedures for animal experiments were reviewed and approved by the Animal Care Committee of the Center of Animal Care and Use at the Lee Gil Ya, Cancer and Diabetes Institute, Gachon University (permit no. LCDI-2013-0022).

Full-thickness wounds and quantification of healing. SKH-1 hairless mice (5 weeks old, male, 25-30 g) were purchased from Shizuoka Laboratory Animal Center and housed in a temperature-controlled room $\left(23^{\circ} \mathrm{C}\right)$ with $65 \%$ humidity and a 12-h light/dark cycle. A total of 40 mice were randomly divided into four groups: Vehicle $(n=10) ; 0.05 \%$ NED416 $(n=10) ; 0.1 \%$ NED416 $(n=10)$; and $0.5 \%$ NED416 $(n=10)$. Two identical wounds were created on the posterior dorsal region of each mouse using a 6-mm biopsy punch. NED416 solutions 
A

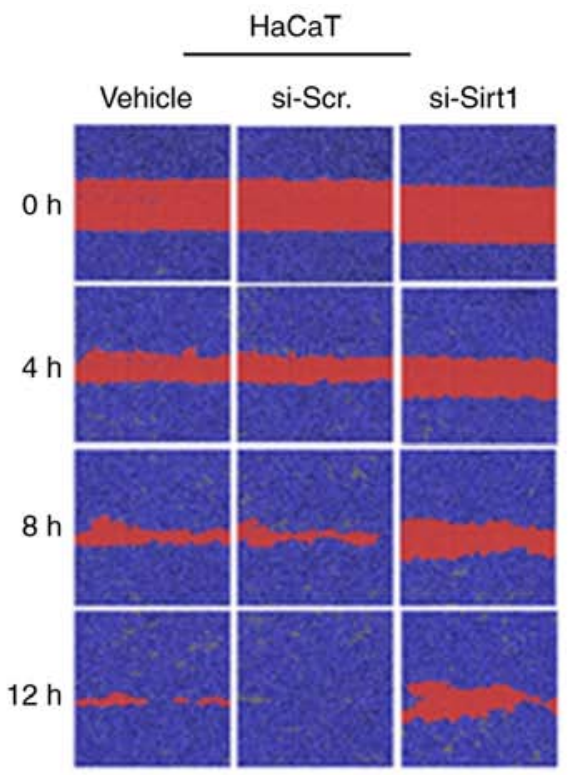

D

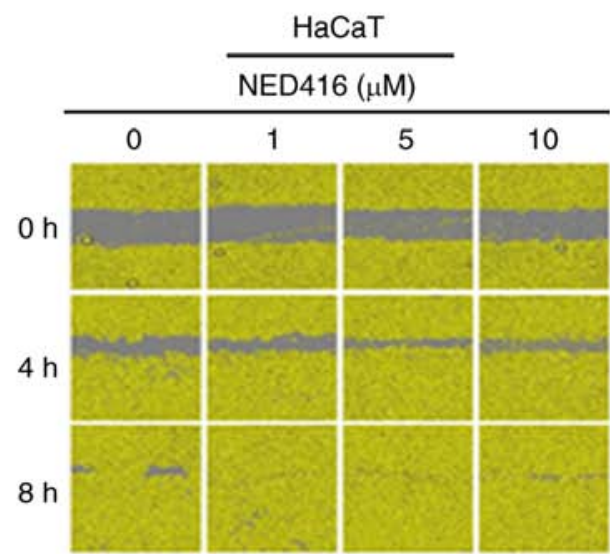

F
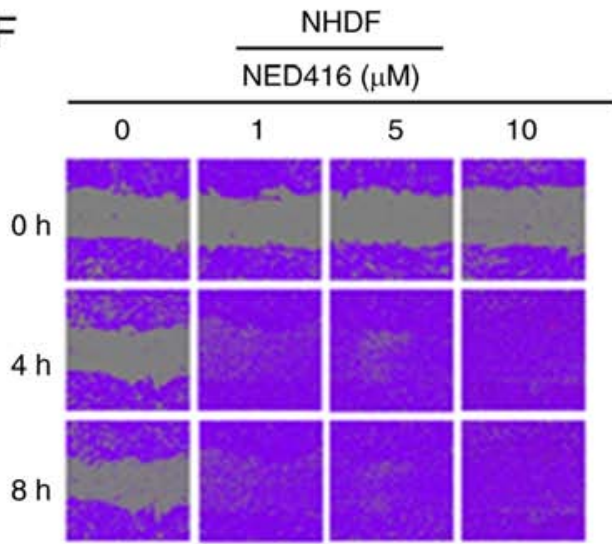

B

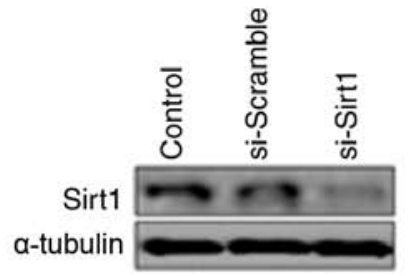

C

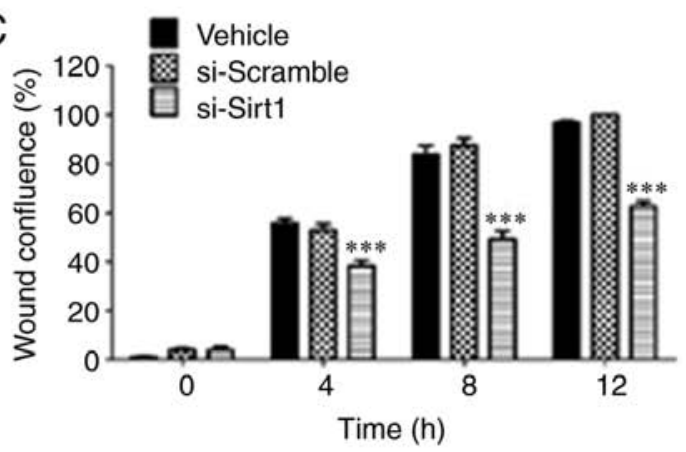

$\mathrm{E}$

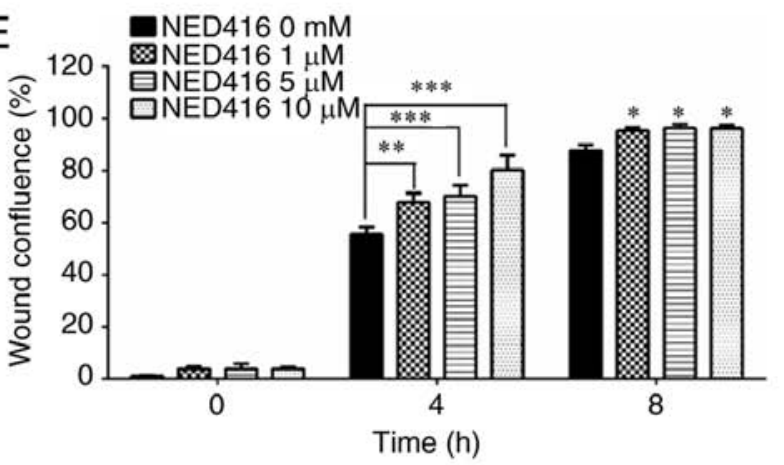

G

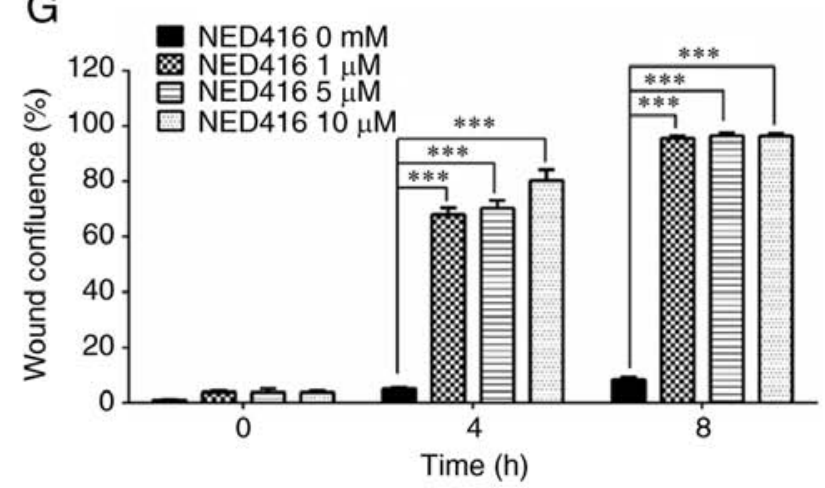

Figure 2. NED416 promotes skin cell migration. (A) Healing of wounded HaCaT cell monolayers following Sirt1 siRNA-mediated knockdown resulted in a slower rate of migration than control or scramble siRNA-treated cells. (B) Western blot showing siRNA knockdown of Sirt1. (C) Quantification of the average wound confluence in control, scramble and Sirt1 siRNA-treated HaCaT cells. ${ }^{* * *} \mathrm{P}<0.001$ vs. control. (D) HaCaT cells cultured in the presence of medium only or NED416 after wounding showed that NED416 treatment increases cell migration in a concentration-dependent manner. (E) Quantification of wound confluence in control and NED416-treated HaCaT cells. (F) NHDF cells cultured in the presence of medium only or NED416 after wounding revealed that $5 \mu \mathrm{M}$ NED416 induced the most pronounced wound healing, followed by 1 and $10 \mu \mathrm{M}$ NED416. (G) Quantification of wound confluence in control and NED416-treated NHDF cells. Data are presented as the mean \pm SEM. Magnification, $\mathrm{x} 40$ of images. ${ }^{*} \mathrm{P}<0.05,{ }^{* *} \mathrm{P}<0.01,{ }^{* * *} \mathrm{P}<0.001$ vs. 0 mM. NED416, (E)-3-(2,4-dichlorophenyl)-N-phenylacrylamide; Sirt1, sirtuin 1; NHDF, normal human dermal fibroblasts; si(RNA), small interfering (RNA).

$(0.05,0.1$ and $0.5 \%)$ dissolved in a mixture of distilled water, ethanol and propanediol (5:3:2, respectively) were applied topically to the wound every day for 10 days. The vehicle group was treated with solvent only.
Histological analysis. Skin samples containing the central part of the wound were collected on days 3, 5, 7 and 10 after wound induction. Specimens were fixed in $10 \%$ formalin for $18 \mathrm{~h}$ at room temperature. Fixed tissues were subjected 

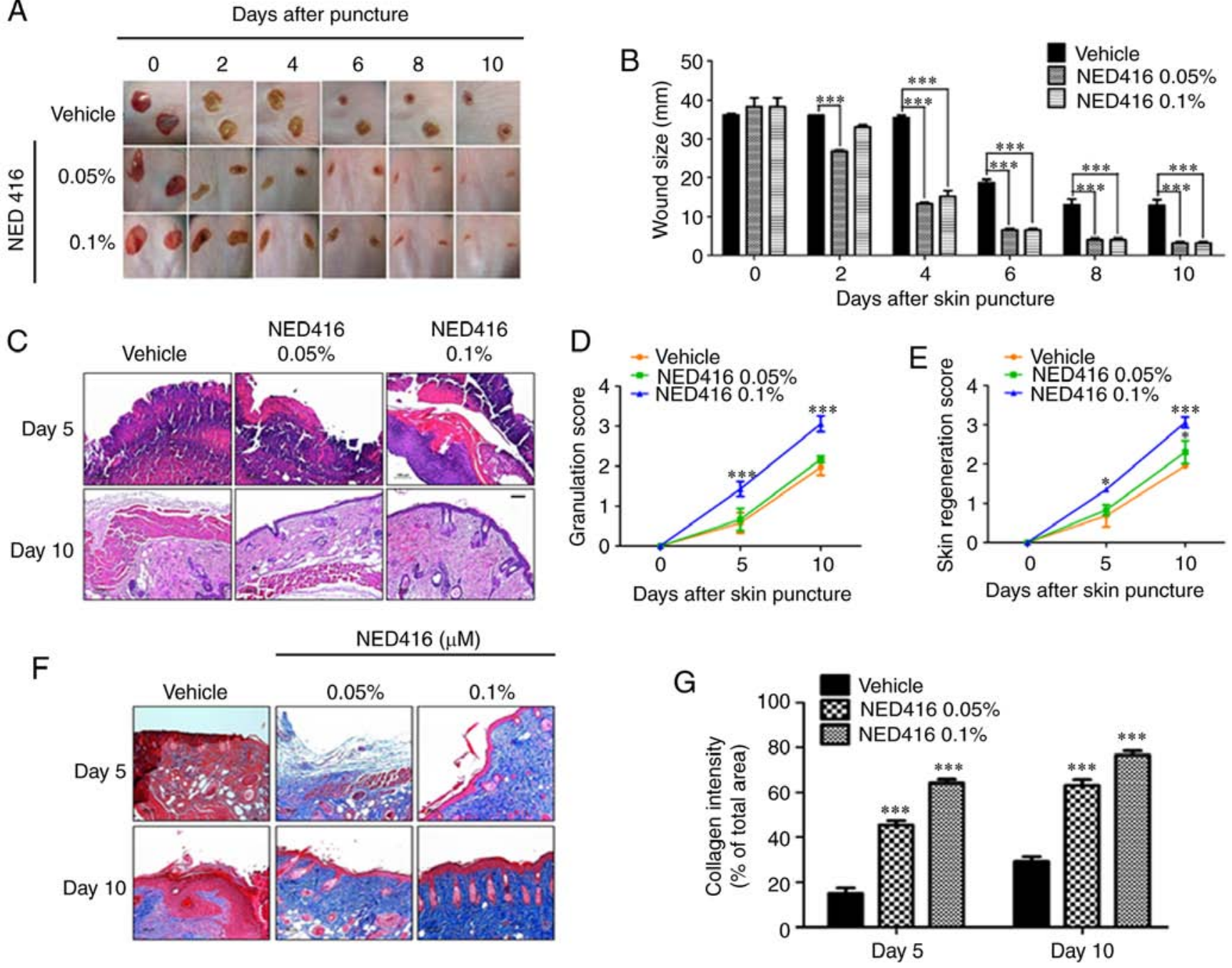

Figure 3. NED416 promotes wound healing and tissue development in mouse skin. (A) Representative images of wounds from each group over 10 days after wounding revealed a faster rate of closure in NED416-treated mice compared with in vehicle-treated mice. (B) Quantification of the average wound area at different time points after injury. (C) Neo-epithelium regeneration in skin collected on days 5 and 10 post-injury monitored by H\&E staining. Magnification, x10. (D) Epidermal and dermal regeneration plotted as histological scores in tissue samples on days 5 and 10 post-injury. Histological score of epidermal and dermal regeneration on days 5 and 10 post-wounding. (E) Histological scores of granulation thickness on days 5 and 10 post-wounding. (F) Skin sections stained with Masson's trichrome show higher levels of collagen in NED416-treated mice at days 5 and 10 post-wounding than in vehicle-treated mice. Magnification, $x 10$. (G) Quantification of collagen deposition in mouse skin on days 5 and 10 post-injury. Data are presented as the mean \pm SEM ( $\mathrm{n}=10 / \mathrm{group}$ ). ${ }^{*} \mathrm{P}<0.05,{ }^{* * * *} \mathrm{P}<0.001$ vs. vehicle. NED416, (E)-3-(2,4-dichlorophenyl)- $N$-phenylacrylamide.

to H\&E and Masson's Trichrome staining as described by Wahedi et al (27). Images were captured using an inverted microscope (magnification, x10). For epidermal regeneration and granulation, a semi-quantitative score system was used as described in Table I (30).

Statistical analysis. Differences between groups were determined using one-way ANOVA with Dunnett's test or Tukey's test as a post hoc test. $\mathrm{P}<0.05$ was considered to indicate a statistically significant difference. The results are presented as the mean \pm standard error of the mean from three independent experiments. Statistical analysis was performed using GraphPad Prism version 6.01 (GraphPad Software, Inc.).

\section{Results}

NED416 increases the activity and protein expression of Sirt1. The effect of NED416 on Sirtl activity was analyzed using an enzymatic activity assay. It was shown that NED416 enhanced the enzymatic activity of Sirt1 in a concentration-dependent manner. This effect of NED416 on Sirt1 activity was greater than that of resveratrol, a well-known Sirt1 activator (Fig. 1A). Western blot analysis showed that NED416 treatment resulted in a concentration-dependent increase in the deacetylation of p53, validating the assay results (Fig. 1B).

NED416 treatment increased Sirtl expression in NHDFs in a concentration-dependent manner (Fig. 1C and D). A similar concentration-dependent effect of NED416 was observed on Sirt1 expression in HaCaT cells (Fig. 1E and F). An MTT assay showed that NED416 treatment did not significantly decrease NHDF or HaCaT cell viability (Fig. S3).

NED416 promotes skin cell migration. To investigate the role of Sirt1 in the migration of HaCaT cells, Sirt1 knockdown was performed, followed by a migration assay in $\mathrm{HaCaT}$ cells. Cells treated with Sirt1 siRNA exhibited a slower rate of 
A

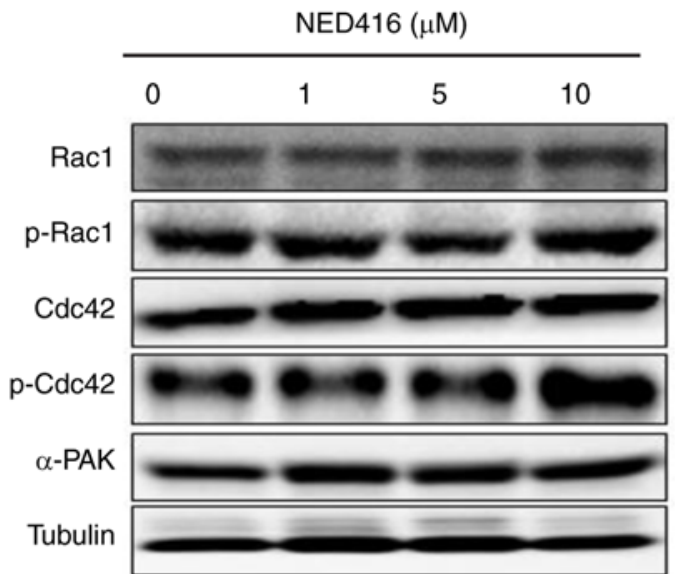

C

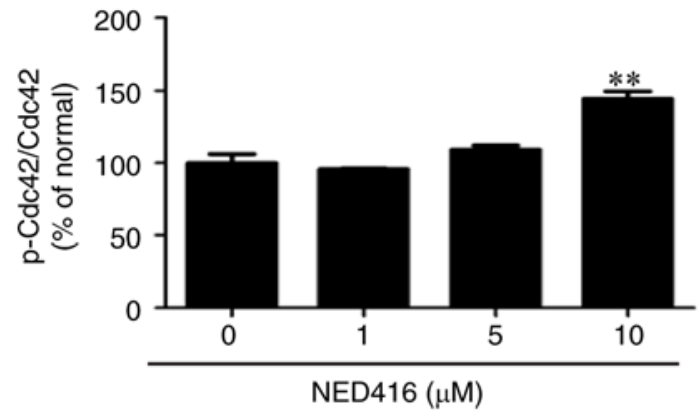

E

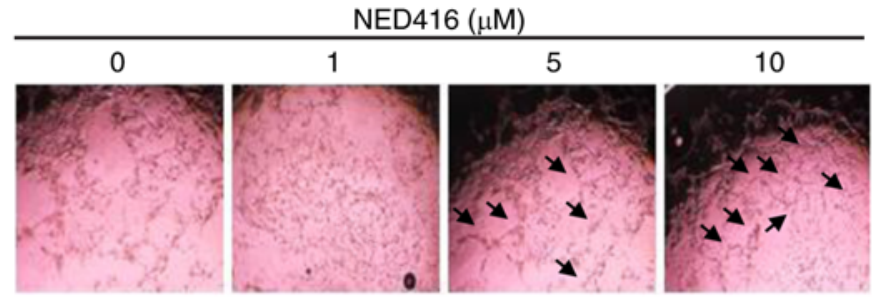

B

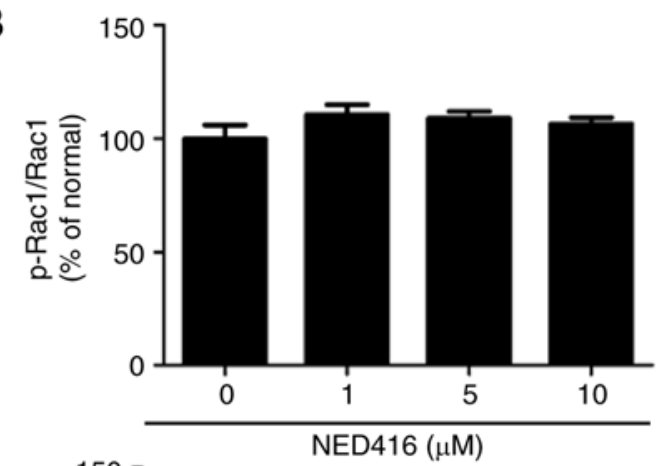

D

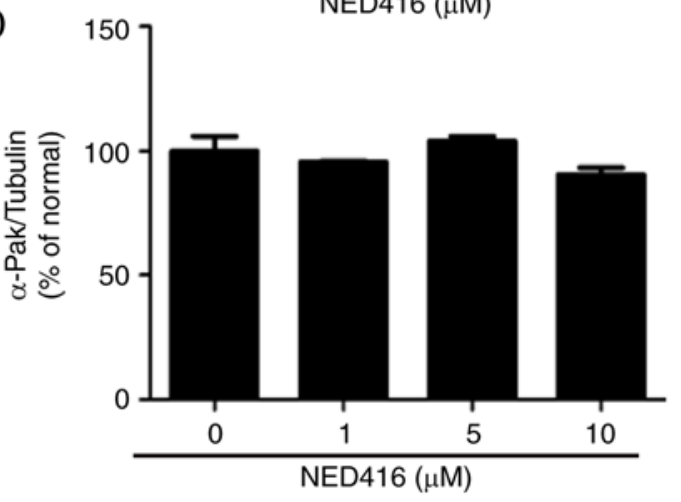

$\mathrm{F}$

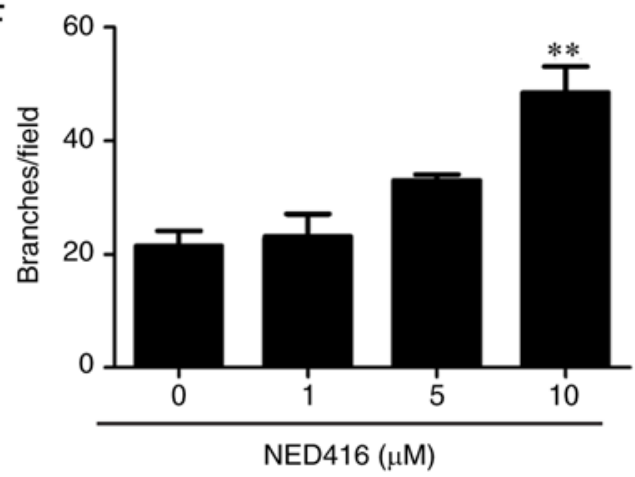

Figure 4. NED416 activates migration-related proteins in HaCaT cells and promotes angiogenesis. (A) Cellular levels of migration-related proteins, including Rac1, Cdc42 and their phosphorylated forms, as well as $\alpha$-Pak, upon treatment with NED416 in HaCaT cells. Quantification of the expression profiles of (B) p-Rac1, (C) p-Cdc42 and (D) $\alpha$-Pak. (E) Microscopic images of HUVECs indicating tube formation after treatment with NED416 and medium only. Magnification, x40. (F) Quantification of the number of branches in HUVECs after $18 \mathrm{~h}$ of NED416 treatment. Data are presented as the mean \pm SEM. ${ }^{* *} \mathrm{P}<0.01$ vs. 0 mM. NED416, (E)-3-(2,4-dichlorophenyl)- $N$-phenylacrylamide; p, phosphorylated; Cdc 42 , cell division cycle $42 ; \alpha$-PAK, $\alpha$-p21-activated kinase; HUVEC, human umbilical vein endothelial cell.

migration than the scramble siRNA-transfected and untreated cells (Fig. 2A and B). These differences in the rates of migration were significant at 4, 8 and $12 \mathrm{~h}$ after treatment (Fig. 2C).

Moreover, cells grown in the presence of NED416 exhibited faster growth rates than the cells grown in the absence of NED416 (Fig. 2D). Treatment with NED416 resulted in faster growth and migration rates, which were concentration-dependent, than those of untreated cells. This difference in the rate of migration was significant at 4 and 8 h (Fig. 2E). NHDF cells grown in the presence of NED416 also exhibited faster growth rates than the cells grown in the absence of NED416, as observed in HaCaT cells (Fig. 2F). These differences in the rates of migration were significant at 4 and $8 \mathrm{~h}$ (Fig. 2G).

NED416 promotes wound healing and tissue regeneration in mouse skin. NED416 was investigated for wound healing potential in a hairless mouse model. According to digital photographs, hairless mice treated with different concentrations of NED416 showed faster wound closure and dermal regeneration than vehicle-treated mice (Fig. 3A). Up to day 2 of injury, wound size was not significantly different between the vehicle and $0.1 \%$ NED groups; however, from day 3 onward, the difference was significant for both concentrations of NED416 (Fig. 3B). NED416-treated mice showed complete wound healing after 8 days, while the wounds in vehicle-treated mice were not healed completely even after 10 days.

Skin tissues were stained with $H \& E$ to monitor tissue development. The skin tissue of NED416-treated mice showed faster and more complete development of granulation tissue and neoepithelium when compared with the skin tissue of untreated mice (Fig. 3C). Based on the criteria presented in Table I, improved dermal and epidermal regeneration was found in NED416-treated mice, along with improved 
A

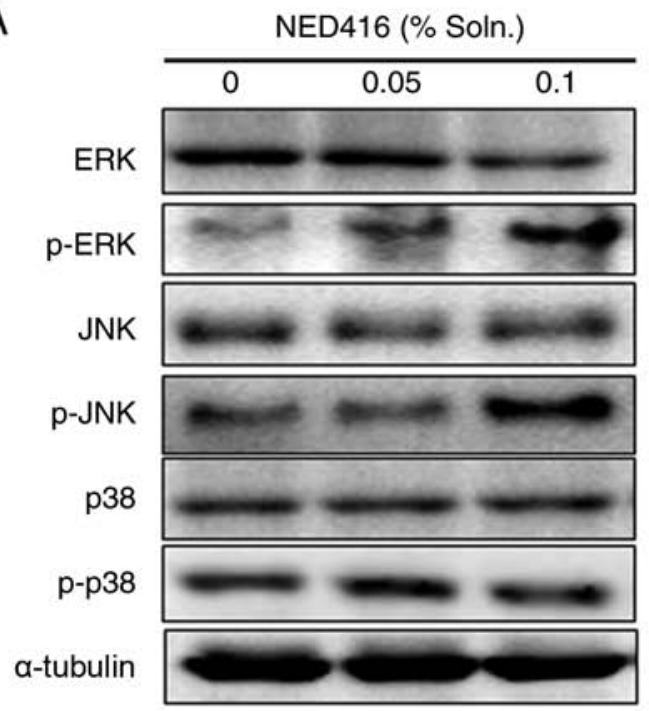

C

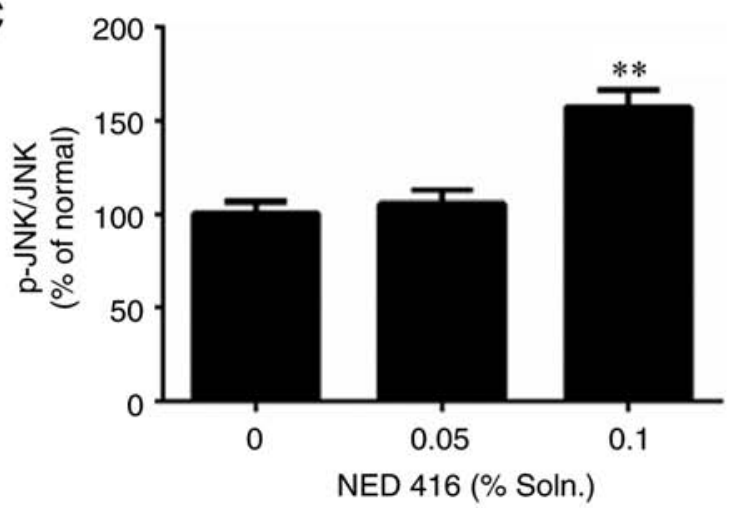

B
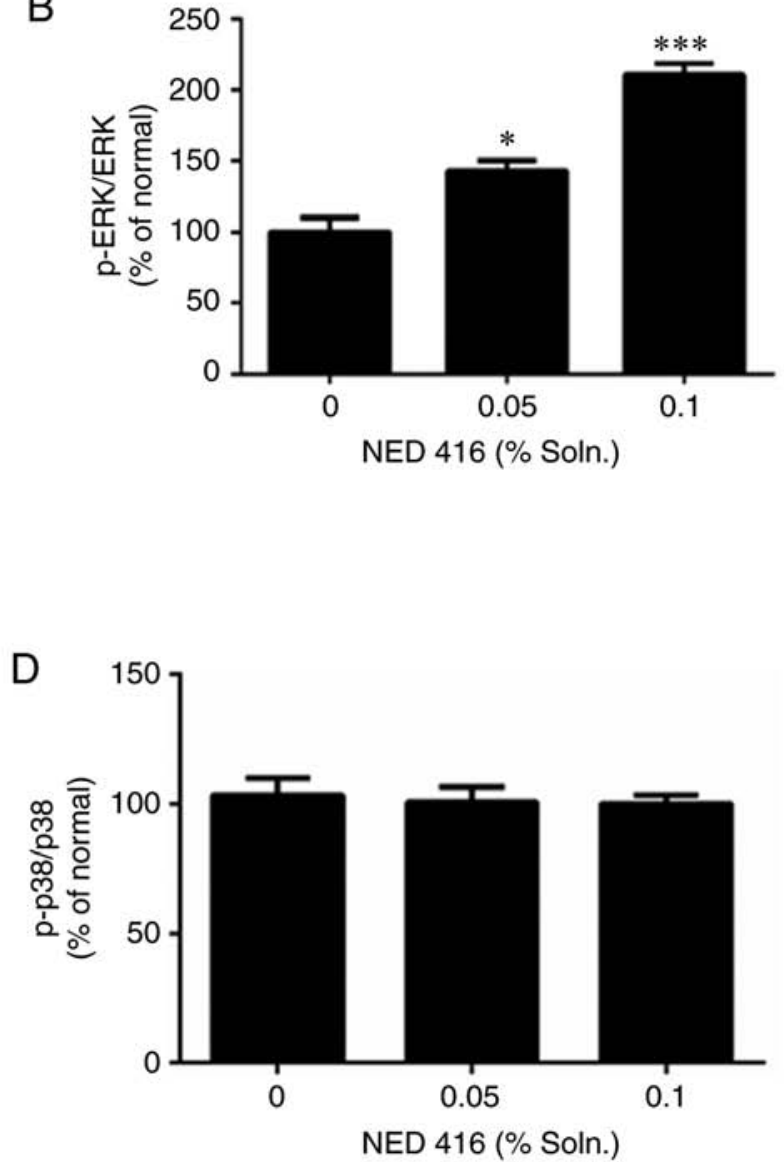

Figure 5. NED416 activates mitogen-activated protein kinase signaling proteins. (A) Expression of p-ERK, p-JNK and p-p38 protein kinases after NED416 treatment in mouse skin samples. Quantification of the activation of (B) ERK, (C) JNK and (D) and p38 upon NED416 treatment. Data are presented as the mean \pm SEM. ${ }^{*} \mathrm{P}<0.05,{ }^{* *} \mathrm{P}<0.01,{ }^{* * *} \mathrm{P}<0.001$ vs. $0 \%$. NED416, $(E)-3$-(2,4-dichlorophenyl)- $N$-phenylacrylamide; p, phosphorylated.

granulation tissue formation, compared with in vehicle-treated mice (Fig. 3D and E). Moreover, the deposition and organization of collagen improved in the skin tissue from the mice treated with NED416 than in those from the mice treated with vehicle only (Fig. 3F and G).

NED416 activates migration-related proteins and angiogenesis. To confirm the finding that NED416 positively affects skin cell migration, the expression and phosphorylation of cell migration-related marker proteins (Rac1, Cdc42 and $\alpha$-Pak) were measured after NED416 treatment of HaCaT cells (Fig. 4A). Western blot analysis showed that expression of Rac1 was not altered by NED416 (Fig. 4B). However, the GTP-bound active form of $\mathrm{Cdc} 42$ was upregulated by NED416 treatment (Fig. 4C), indicating the activation of $\mathrm{Cdc} 42$. The expression of $\alpha$-Pak was also not altered by NED416 treatment (Fig. 4D).

To measure the effect of NED416 on angiogenesis, HUVECs cultured with NED416 and medium only were photographed to monitor the progress of tube formation. Microscopic images captured $16 \mathrm{~h}$ post-treatment showed that cells treated with NED416 exhibited notably enhanced angiogenesis, in that the number of branches in these cells was greater than in untreated cells (Fig. 4E). Although the effect of
NED416 was only significant at $10 \mu \mathrm{M}$, a notable trend towards increased angiogenesis was also observed in $5 \mu \mathrm{M}$-treated cells (Fig. 4F).

NED416 activates mitogen-activated protein kinase (MAPK) signaling proteins. The expression of MAPKs was evaluated, as they are intracellular signaling molecules that may interact with Rho family proteins to mediate cell migration (31). As determined via western blot analysis, skin samples treated with NED416 exhibited increases in ERK and JNK activation (Fig. 5A), with significantly increased relative levels of p-ERK1/2 (Fig. 5B) and p-JNK compared with the untreated tissues (Fig. 5C). However, NED416 did not affect the expression of p-p38 (Fig. 5D). To confirm the role of MAPK signaling in increased cell migration, ERK, JNK and p38 were blocked by using their specific inhibitors in the presence of NED416 in cultured HaCaT cells. Each of the inhibitors reduced NED416-mediated skin cell migration (Fig. S4).

\section{Discussion}

The present study aimed to reveal the effects of NED416 on Sirtl activation and thus on the process of cutaneous wound healing based on the fact that Sirt1 and its regulators have been 
shown to play a role in cell migration and tissue healing $(24,25)$. The ability of NED416 to increase Sirt1 activity, as well as Sirtl expression, in NHDF and HaCaT cells indicated that it is a Sirt1 activator. NED416 promoted the cellular levels of Sirt1 and enhanced its enzymatic activity. At present, isoflavones and resveratrol are the most widely known natural compounds that can increase both the protein expression and activity of Sirt1 (32); most synthetic regulators of Sirt1 only alter the activity of Sirt1 $(33,34)$. NED416 not only enhanced Sirt1 activity over that of control cells but was more effective than resveratrol, which is an established Sirt1 activator (35). The MTT assay for NED416 also showed that NED416 was not toxic at effective concentrations in NHDF or HaCaT cells. The ability of NED416 to increase Sirt1 activity and expression over that of resveratrol suggests that its mode of action differs from that of resveratrol and may provide a diverse approach to targeting Sirt1.

Knocking down Sirt1 expression in skin keratinocytes resulted in decreased migration, suggesting that Sirt1 may play a role in cell migration during wound healing. Treatment with NED416 promoted the rate of migration in HaCaT cells. Differences in migration rates were significant at all time points between NED416-treated and untreated cells. Similar results were found when the same experiment was performed using NHDFs. Both skin fibroblasts and keratinocytes were included in the study as they are involved in rejuvenation and wound healing $(9,36)$. Migration assays using NHDFs and $\mathrm{HaCaT}$ cells revealed increased migration rates in the presence of NED416, proving it an effective Sirt1 activator for its effects on skin cell migration and pointing toward the involvement of Sirt1 in cell migration during wound healing. These findings are consistent with the results of previous studies of the involvement of Sirt1 and its activators in tissue healing and cell migration $(24,25,27)$.

To confirm the in vitro effects of NED416 in an animal model, its effects on cutaneous wound healing were tested in hairless mice. As hypothesized, the differences in wound healing were significant at all time points from day 2 onward for animals treated with NED416 compared with vehicle. Skin samples from the NED416-treated mice exhibited faster neoepithelium, dermal and epidermal regeneration when subjected to H\&E staining. These results not only complemented the results found in cultured keratinocyte and fibroblast monolayers, but also offered insight into epithelial migration facilitated by NED416 in injured skin tissue. Furthermore, these findings demonstrated the wound healing potential of NED416 in vivo as re-epithelialization is one of the most critical and complex steps of wound healing (1). Thus, in addition to later stages such as migration and tissue remodeling, NED416 positively regulated the early stages of wound closure and inflammation. Resveratrol and juglone are Sirt1 activators from plant sources that showed wound healing potential in previous studies $(27,37,38)$. Sirt1 activators, including NED416, regulate wound healing mainly in the later phases. However, extracts from Aloe vera and Vitis vinifera regulate the overall process of wound healing $(38,39)$.

NED416 increased the quantity of collagen present in skin tissue samples that were collected 5 and 10 days after injury. The amounts of collagen deposited were also significantly higher in the NED416-treated groups than in the vehicle-treated group. This finding indicated that NED416 promoted the generation and deposition of collagen, a marker of the remodeling phase, and is consistent with the results of previous studies in which collagen fibers and elastin indicate the replacement and rearrangement of old and disorganized collagen $(7,40)$.

To confirm the wound healing effects of NED416, the expression and phosphorylation levels of migration-related proteins (Rac1, Cdc42 and $\alpha$-Pak) were monitored, as these proteins play important roles in cell migration and thus the promotion of wound healing (10-12). Protein expression of Cdc42 induced by NED416 treatment was significantly increased. The expression of Rac1 was not different between its phosphorylated and unphosphorylated forms upon treatment with NED416. However, the relative expression of p-Cdc42 compared with its unphosphorylated form was increased following treatment with NED416. The expression of $\alpha$-Pak was also not markedly increased. These findings showed that NED416 promoted wound healing by enhancing skin cell migration via the Rac1/Cdc42 pathway. The activation of $\mathrm{Cdc} 42$ is significant in the sense that activation of Rac1 depends on $\mathrm{Cdc} 42$, and $\alpha$-Pak is a downstream target of the Cdc42/Rac1 complex (41).

Cdc42 is involved in controlling cell migration through filopodia formation in actin filaments, whereas Rac1 and $\alpha$-Pak control lamellipodia and stress fiber formation, respectively (42-47). Thus, Cdcd42 activation is the most significant and vital event in cell migration induced by NED416 treatment. Neoangiogenesis plays an essential role in the development and nourishment of nascent tissue, and the removal of waste from the wound area (48). The ability of NED416 to promote angiogenesis in cultured HUVECs also supported the wound healing potential of NED416.

Compared with vehicle treatment, NED416 treatment activated MAPK signaling, as western blot analysis revealed increased levels of ERK and JNK in their phosphorylated forms. Of note, NED416 increased p-ERK and p-JNK expression in a dose-dependent manner. However, phosphorylation of p38 was not increased. These findings regarding MAPK signaling pathway activation by NED416 are of note as cell migration has been shown to involve the ERK and JNK signaling pathways to promote the process of wound healing $(49,50)$.

In conclusion, NED416 is a novel synthetic Sirt1 activator that can increase Sirt1 activity more than resveratrol, and ameliorates the overall process of wound healing in cultured skin cells and a mouse wound model, mainly in the later processes of cell migration, angiogenesis and tissue remodeling. NED416 promotes these processes by regulating the Rac1/Cdc42 and MAPK signaling pathways. Future studies with NED416 and other Sirt1 activators not only have the potential for developing new therapeutic strategies for wound healing, but may also lead to a novel paradigm of research to reveal the role of Sirt1 in cutaneous wound healing, which is one of the most complex and essential repair and regeneration processes.

\section{Acknowledgments}

We acknowledge Professor Tae Yoon Kim of the College of Medicine of The Catholic University of Korea for providing the HaCaT cell line. 


\section{Funding}

This study was supported by a grant from the Korean Health Technology R\&D Project, Ministry of Health \& Welfare, Republic of Korea (grant no. HN10C0017).

\section{Availability of data and materials}

The datasets used and/or analyzed during the current study are available from the corresponding author on reasonable request.

\section{Authors' contributions}

HMW performed the experiments, analyzed the data and prepared the manuscript. JKC, LS and MCK performed experiments. HC and SK performed the compound synthesis. SYK conceived, designed and supervised the study. All authors read and approved the final manuscript.

\section{Ethics approval and consent to participate}

All procedures for animal experiments were reviewed and approved by the Animal Care Committee of the Center of Animal Care and Use at the Lee Gil Ya, Cancer and Diabetes Institute, Gachon University (permit no. LCDI-2013-0022).

\section{Patient consent for participation}

Not applicable.

\section{Competing interests}

The authors declare that they have no competing interests.

\section{References}

1. Gurtner GC, Werner S, Barrandon Y and Longaker MT: Wound repair and regeneration. Nature 453: 314-321, 2008.

2. Li J, Chen J and Kirsner R: Pathophysiology of acute wound healing. Clin Dermatol 25: 9-18, 2007.

3. Kondo T and Ishida Y: Molecular pathology of wound healing. Forensic Sci Int 203: 93-98, 2010.

4. Reinke JM and Sorg H: Wound repair and regeneration. Eur Surg Res 49: 35-43, 2012.

5. Singer AJ and Clark RA: Cutaneous wound healing. N Engl J Med 341: 738-746, 1999.

6. Werner S and Grose R: Regulation of wound healing by growth factors and cytokines. Physiol Rev 83: 835-870, 2003.

7. Hantash BM, Zhao L, Knowles JA and Lorenz HP: Adult and fetal wound healing. Front Biosci 13: 51-61, 2008.

8. Raja, Sivamani K, Garcia MS and Isseroff RR: Wound re-epithelialization: Modulating keratinocyte migration in wound healing. Front Biosci 12: 2849-2868, 2007.

9. Wojtowicz AM, Oliveira S, Carlson MW, Zawadzka A, Rousseau CF and Baksh D: The importance of both fibroblasts and keratinocytes in a bilayered living cellular construct used in wound healing. Wound Repair Regen 22: 246-255, 2014.

10. Braun A, Dang K, Buslig F, Baird MA, Davidson MW, Waterman CM and Myers KA: Racl and Aurora A regulate MCAK to polarize microtubule growth in migrating endothelial cells. J Cell Biol 206: 97-112, 2014.

11. Baek SH, Cho HW, Kwon YC, Lee JH, Kim MJ, Lee H and Choe KM: Requirement for Pak3 in Rac1-induced organization of actin and myosin during Drosophila larval wound healing. FEBS Lett 586: 772-777, 2012.
12. Funasaka $\mathrm{K}$, Ito $\mathrm{S}$, Hasegawa $\mathrm{H}$, Goldberg GS, Hirooka $\mathrm{Y}$, Goto H, Hamaguchi $M$ and Senga T: Cas utilizes Nck2 to activate $\mathrm{Cdc} 42$ and regulate cell polarization during cell migration in response to wound healing. FEBS J 277: 3502-3513, 2010.

13. Barrientos S, Stojadinovic O, Golinko MS, Brem H and Tomic-Canic M: Growth factors and cytokines in wound healing. Wound Repair Regen 16: 585-601, 2008.

14. Welch MP, Odland GF and Clark RA: Temporal relationships of F-actin bundle formation, collagen and fibronectin matrix assembly, and fibronectin receptor expression to wound contraction. J Cell Biol 110: 133-145, 1990.

15. Michan S and Sinclair D: Sirtuins in mammals: Insights into their biological function. Biochem J 404: 1-13, 2007.

16. Orecchia A, Scarponi C, Di Felice F, Cesarini E, Avitabile S, Mai A, Mauro ML, Sirri V, Zambruno G, Albanesi C, et al: Sirtinol treatment reduces inflammation in human dermal microvascular endothelial cells. PLoS One 6: e24307, 2011.

17. Liu Y, He XQ, Huang X, Ding L, Xu L, Shen YT, Zhang F, Zhu MB, Xu BH, Qi ZQ and Wang HL: Resveratrol protects mouse oocytes from methylglyoxal-induced oxidative damage. PLoS One 8: e77960, 2013.

18. Yamakuchi M, Ferlito $M$ and Lowenstein CJ: miR-34a repression of SIRT1 regulates apoptosis. Proc Natl Acad Sci USA 105: 13421-13426, 2008.

19. Lee JH, Song MY, Song EK, Kim EK, Moon WS, Han MK, Park JW, Kwon KB and Park BH: Overexpression of SIRT1 protects pancreatic beta-cells against cytokine toxicity by suppressing the nuclear factor-kappaB signaling pathway. Diabetes 58: 344-351, 2009.

20. Satoh A, Brace CS, Rensing N, Cliften P, Wozniak DF, Herzog ED, Yamada KA and Imai S: Sirtl extends life span and delays aging in mice through the regulation of Nk2 homeobox 1 in the DMH and LH. Cell Metabol 18: 416-430, 2013.

21. Valente S, Mellini P, Spallotta F, Carafa V, Nebbioso A, Polletta L, Carnevale I, Saladini S, Trisciuoglio D, Gabellini C, et al: 1,4-Dihydropyridines active on the SIRT1/AMPK pathway ameliorate skin repair and mitochondrial function and exhibit inhibition of proliferation in cancer cells. J Med Chem 59: 1471-1491, 2016.

22. Wang Y, Zhao X, Shi D, Chen P, Yu Y, Yang L and Xie L: Overexpression of SIRT1 promotes high glucose-attenuated corneal epithelial wound healing via 553 regulation of the IGFBP3/IGF-1R/AKT pathway. Invest Ophthalmol Vis Sci 54: 3806-3814, 2013

23. Hunt ND, Li GD, Zhu M, Miller M, Levette A, Chachich ME, Spangler EL, Allard JS, Hyun DH, Ingram DK and de Cabo R: Effect of calorie restriction and refeeding on skin wound healing in the rat. Age (Dordr) 34: 1453-1458, 2012.

24. Zeytin K, Ciloğlu NS, Ateș F, Vardar Aker F and Ercan F: The effects of resveratrol on tendon healing of diabetic rats. Acta Orthop Traumatol Turc 48: 355-362, 2014.

25. Casarin RC, Casati MZ, Pimentel SP, Cirano FR, Algayer M, Pires PR, Ghiraldini B, Duarte PM and Ribeiro FV: Resveratrol improves bone repair by modulation of bone morphogenetic proteins and osteopontin gene expression in rats. Int $\mathbf{J}$ Oral Maxillofac Surg 43: 900-906, 2014.

26. Wahedi HM, Lee TH, Moon EY and Kim SY: Juglone up-regulates sirt1 in skin cells under normal and UVB irradiated conditions. J Dermatol Sci 81: 210-212, 2016.

27. Wahedi HM, Park YU, Moon EY and Kim SY: Juglone ameliorates skin wound healing by promoting skin cell migration through Rac1/Cdc42/PAK pathway. Wound Repair Regen 24: 786-794, 2016.

28. Kim S, Lim C, Lee S, Lee S, Cho H, Lee JY, Shim DS, Park HD and Kim S: Column chromatography-free solution-phase synthesis of a natural piper-amide-like compound library. ACS Comb Sci 15: 208-215, 2013.

29. Qiu J and Zhang R: Direct transformation of arylpropynes to acrylamides via a three-step tandem reaction. Org Biomol Chem 12: 1556-1560, 2014.

30. Galeano M, Deodato B, Altavilla D, Cucinotta D, Arsic N, Marini H, Torre V, Giacca M and Squadrito F: Adeno-associated viral vector-mediated human vascular endothelial growth factor gene transfer stimulates angiogenesis and wound healing in the genetically diabetic mouse. Diabetologia 46: 546-555, 2003.

31. Cheng C, Kong X, Wang H, Gan H, Hao Y, Zou W, Wu J, Chi Y, Yang J, Hong Y, et al: Trihydrophobin 1 interacts with PAK1 and regulates ERK/MAPK activation and cell migration. J Biol Chem 284: 8786-8796, 2009. 
32. Dai H, Sinclair DA, Ellis JL and Steegborn C: Sirtuin activators and inhibitors: Promises, achievements, and challenges. Pharmacol Ther 188: 140-154, 2018.

33. Kumar A and Chauhan S: How much successful are the medicinal chemists in modulation of SIRT1: A critical review. Eur J Med Chem 119: 45-69, 2016.

34. Hubbard BP and Sinclair DA: Small molecule SIRT1 activators for the treatment of aging and age-related diseases. Trends Pharmacol Sci 35: 146-154, 2014.

35. Alcain FJ and Villalba JM: Sirtuin activators. Expert Opin Ther Pat 19: 403-414, 2009.

36. Lee TH, Lee GW, Park KH, Mohamed MA, Bang MH, Baek YS, Son Y, Chung DK, Baek NI and Kim J: The stimulatory effects of Stewartia koreana extract on the proliferation and migration of fibroblasts and the wound healing activity of the extract in mice. Int J Mol Med 34: 145-152, 2014.

37. Amanat S, Taymouri S, Varshosaz J, Minaiyan M and Talebi A: Carboxymethyl cellulose-based wafer enriched with resveratrol-loaded nanoparticles for enhanced wound healing. Drug Deliv Transl Res: Jan 24, 2020 (Epub ahead of print).

38. Lin LX, Wang P, Wang YT, Huang Y, Jiang L and Wang XM: Aloe vera and Vitis vinifera improve wound healing in an in vivo rat burn wound model. Mol Med Rep 13: 1070-1076, 2016.

39. Lodhi S, Jain AP, Rai G and Yadav AK: Preliminary investigation for wound healing and anti-inflammatory effects of Bambusa vulgaris leaves in rats. J Ayurveda Integr Med 7: 14-22, 2016.

40. Hornstra IK, Birge S, Starcher B, Bailey AJ, Mecham RP and Shapiro SD: Lysyl oxidase is required for vascular and diaphragmatic development in mice. J Biol Chem 278: 14387-14393, 2003

41. Manser E, Leung T, Salihuddin H, Zhao ZS and Lim L: A brain serine/threonine protein kinase activated by Cdc42 and Rac1. Nature 367: 40-46, 1994.

42. Wakayama Y,Fukuhara S, Ando K, Matsuda M and Mochizuki N Cdc42 mediates Bmp-induced sprouting angiogenesis through Fmnl3-driven assembly of endothelial filopodia in zebrafish. Dev Cell 32: 109-122, 2015.

43. Reddy PN, Radu M, Xu K, Wood J, Harris CE, Chernoff J and Williams DA: p21-activated kinase 2 regulates HSPC cytoskeleton, migration, and homing via CDC42 activation and interaction with beta-Pix. Blood 127: 1967-1975, 2016.
44. Abraham S, Scarcia M, Bagshaw RD, McMahon K, Grant G, Harvey T, Yeo M, Esteves FOG, Thygesen HH, Jones PF, et al: A Rac/Cdc42 exchange factor complex promotes formation of lateral filopodia and blood vessel lumen morphogenesis. Nat Commun 6: 7286, 2015 .

45. King SJ, Asokan SB, Haynes EM, Zimmerman SP, Rotty JD, Alb JG Jr, Tagliatela A, Blake DR, Lebedeva IP, Marston D, et al: Lamellipodia are crucial for haptotactic sensing and response. J Cell Sci 129: 2329-2342, 2016.

46. Gujdar A, Sipeki S, Bander E, Buday L and Faragó A: Phorbol ester-induced migration of HepG2 cells is accompanied by intensive stress fibre formation, enhanced integrin expression and transient down-regulation of p21-activated kinase 1. Cell Signal 15: 307-318, 2003.

47. Kundumani-Sridharan V, Singh NK, Kumar S, Gadepalli R and Rao GN: Nuclear factor of activated T cells c1 mediates p21-activated kinase 1 activation in the modulation of chemokine-induced human aortic smooth muscle cell F-actin stress fiber formation, migration, and proliferation and injury-induced vascular wall remodeling. J Biol Chem 288: 22150-22162, 2013.

48. Carmeliet P: Angiogenesis in health and disease. Nat Med 9: 653-660, 2003.

49. Zhang M, Sun L, Wang X, Chen S, Kong Y, Liu N, Chen Y, Jia Q, Zhang L and Zhang L: Activin B promotes BMSC-mediated cutaneous wound healing by regulating cell migration via the JNK-ERK signaling pathway. Cell Transplant 23: 1061-1073, 2014.

50. Zhang L, Deng M, Parthasarathy R, Wang L, Mongan M, Molkentin JD, Zheng Y and Xia Y: MEKK1 transduces activin signals in keratinocytes to induce actin stress fiber formation and migration. Mol Cell Biol 25: 60-65, 2005.

This work is licensed under a Creative Commons Attribution-NonCommercial-NoDerivatives 4.0 International (CC BY-NC-ND 4.0) License. 